\title{
Coefficient Inequalities for Certain New Subclass of Analytic Univalent Functions in the Unit Disk
}

\author{
${ }^{1}$ Hamzat J. O, ${ }^{2}$ Gbolagade A.M \\ ${ }^{I}$ Department of Pure and Applied Mathematics,Ladoke Akintola University of Technology,P.M.B. 4000, \\ Ogbomoso, Oyo State, Nigeria. \\ ${ }^{2}$ Department of Mathematics, Emmanuel Alayande College of Education,P.M.B. 1010, Oyo, Oyo State, Nigeria.
}

Abstract: For functions $f(z)$ of the form:

$$
f(z)=z+\sum_{k=2}^{\infty} a_{k} z^{k}
$$

which are starlike and convex of order $\delta$ in the open unit disk $U$, the authors derive a new subclass of normalized analytic functions in the open unit $U$. The results presented in this paper generalize many existing results in the literature.

Mathematics Subject Classification: Primary 30C45

Keywords: Analytic, Univalent, Close-to-convex, Starlike, Convex, Coefficient inequalities

\section{Introduction}

Let $J$ be the class of normalized analytic functions of the from

$$
f(z)=z+\sum_{k=2}^{\infty} a_{k} z^{k}
$$

in the open unit disk $U=\{z:|z|<1\}$.

We denote by $S$ the subclass of $J$, consisting of functions which are also univalent in $U$.

Here, we recall the famous classes of starlike and convex functions:

$$
\begin{gathered}
S^{*}=\left\{f \in J: \operatorname{Re}\left(\frac{z f^{\prime}(z)}{f(z)}\right)>0, \quad z \in U\right\}, \\
S^{c}=\left\{f \in J: \operatorname{Re}\left(1+\frac{z f^{\prime \prime}(z)}{f^{\prime}(z)}\right)>0, \quad z \in U\right\} .
\end{gathered}
$$

Let $\omega$ be a fixed point in $U$ and

$$
J(\omega)=\left\{f \in H(U): f(\omega)=f^{\prime}(\omega)-1=0\right\} .
$$

In [11], Kanas and Ronning introduced the following classes

$$
\begin{gathered}
S_{\omega}=\{f \in J(\omega): f \text { is univalent in } U\} \\
S T_{\omega}=\left\{f \in J(\omega): \mathfrak{R}\left(\frac{(z-\omega) f^{\prime}(z)}{f(z)}\right)>0, z \in U\right\}, \\
C V_{\omega}=\left\{f \in J(\omega): \mathfrak{R}\left(1+\frac{(z-\omega) f^{\prime \prime}(z)}{f^{\prime \prime}(z)}\right)>0, z \in U\right\}
\end{gathered}
$$

and these classes have been studied extensively by Acu and Owa [1]. We recall that a region of the complex plane is said to have star geometry with respect to a fixed point in it if every other points of it is visible from the fixed point. In other words, a ray or line segment issuing from the fixed point inside the region to any other point of it lies entirely in the region. Also, if a region has star geometry with respect to every point in it, it is called convex. That is, the line segment joining any two points of this region lies entirely inside it. Therefore, the class $S_{\omega}^{*}$ is defined by geometric property that the image of any circular arc centered at $\omega$ is starlike with respect to $f(\omega)$ and the corresponding class $S_{\omega}^{c}$ is defined by the property that the image of any circular arc 
centered at $\omega$ is convex. Thus, we observe that the definitions are similar to the ones introduced by Goodman in [8] and [9] for uniformly starlike and convex functions, except that in this case the point $\omega$ is fixed.

The function $f(z)$ in $S_{\omega}$ is said to be starlike of order $\delta$ if and only if

$$
\mathfrak{R}\left(\frac{(z-\omega) f^{\prime}(z)}{f(z)}\right)>\delta z \in U
$$

for some $\delta(0 \leq \delta<1)$. We denote by $S T_{\omega}(\delta)$ the class of all starlike functions of order $\delta$. Similarly, a function $f(z)$ in $S_{\omega}$ is said to be convex of order $\delta$ if and only if

$$
\mathfrak{R}\left\{1+\frac{(z-\omega) f^{\prime \prime}(z)}{f^{\prime}(z)}\right\}>\delta \quad z \in U
$$

for some $\delta(0 \leq \delta<1)$. We denote by $C V_{\omega}(\delta)$ the class of all convex function of order $\delta$. We note that the class $S T_{0}(\delta)$ and various other subclasses of $S T_{\omega}(\delta)$ have been widely studied by many authors (see [Acu and Owa[1], Aouf[2], Cho et al[3], Clunie[4], Duren[5], Goel and Sohi[7], Ghanim et al[10], Oladipo[12] and Uralgaddi and Somanatha[13] ) to mention just a few.

Let $S_{\omega}(p)$ denote the subclasses of $J(\omega)$ consisting of the function of the from

$$
f(z)=\frac{\alpha}{(z-\omega)^{p}}+\sum_{k=1}^{\infty} a_{k}(z-\omega)^{k} \quad\left(a_{K} \geq 0\right)
$$

where $\alpha=\operatorname{Re} s(z, \omega)$ with $0<\alpha \leq 1$. For the function $f(z)$ in the class $S_{\omega}(p)$, we define

$$
\begin{gathered}
I_{p}^{0} f(z)=f(z) \\
I_{p}^{1} f(z)=(z-\omega) f^{\prime}(z)+\frac{2 \alpha p}{(\alpha-\omega)^{p}}=\frac{\alpha p}{(\alpha-\omega)^{p}}+\sum_{k=1}^{\infty} k a_{k}(z-\omega)^{k} \\
I_{p}^{2} f(z)=(z-\omega)\left(I_{p}^{1} f(z)\right)^{\prime}+\frac{2 \alpha p^{2}}{(\alpha-\omega)^{p}}=\frac{2 \alpha p^{2}}{(\alpha-\omega)^{p}}+\sum_{k=1}^{\infty} k^{2} a_{k}(z-\omega)^{k}
\end{gathered}
$$

and for $n=1,2,3 \ldots$, we can write

Now, we let,

$$
I_{p}^{n} f(z)=(z-\omega)\left(I_{p}^{n-1} f(z)\right)^{\prime}+\frac{2 \alpha p^{n}}{(\alpha-\omega)^{p}}=\frac{\alpha p^{n}}{(\alpha-\omega)^{p}}+\sum_{k=1}^{\infty} k^{n} a_{k}(z-\omega)^{k} .
$$

That is,

$$
f(z)^{\beta}=\left[\frac{\alpha}{(z-\omega)^{p}}+\sum_{k=1}^{\infty} a_{k}(z-\omega)^{k}\right]^{\beta} .
$$

$$
\begin{gathered}
f(z)^{\beta}=\alpha^{\beta}(z-\omega)^{-p \beta}\left[1+\frac{\beta}{\alpha}\left(a_{1}(z-\omega)^{p+1}+a_{2}(z-\omega)^{p+2}+\ldots\right)\right. \\
\left.+\frac{\beta(\beta-1)}{2 ! \alpha^{2}}\left(a_{1}(z-\omega)^{p+1}+a_{2}(z-\omega)^{p+2}+\ldots\right)^{2}+\ldots\right] \\
=(z-\omega)^{-p \beta}\left\lfloor\alpha^{\beta}+\beta \alpha^{\beta-1}\left(a_{1}(z-\omega)^{p-1}+a_{2}(z-\omega)^{p+2}+\ldots\right)\right. \\
\left.+\frac{\beta(\beta-1)}{2 !} \alpha^{\beta-2}\left(a_{1}(z-\omega)^{p+1}+a_{2}(z-\omega)^{p+2}+\ldots\right)^{2}+\ldots\right]
\end{gathered}
$$

which implies that

where,

$$
f(z)^{\beta}=(z-\omega)^{-p \beta}\left[\alpha^{\beta}+\sum_{m=1}^{\infty} \beta_{m}\left(a_{1}(z-\omega)^{p+1}+a_{2}(z-\omega)^{p+2}+\ldots\right)^{m}\right]
$$


Therefore,

$$
\beta_{1}=\beta \alpha^{\beta-1}, \beta_{2}=\frac{\beta(\beta-1)}{2 !} \alpha^{\beta-2}, \ldots
$$

$$
f(z)^{\beta}=\frac{\alpha^{\beta}}{(z-\omega)^{p \beta}}+\sum_{k=1}^{\infty} a_{k}(\beta)(z-\omega)^{k+p(1-\beta)}
$$

where $\alpha=\operatorname{Re} s(z-\omega)$ with $0<\alpha \leq 1$ and $p, \beta \in N$.

For function $f(z)^{\beta}$ in the class $S_{\omega}(p, \beta)$, we define

$$
\begin{gathered}
I_{p}^{0} f(z)=f(z)^{\beta} \\
I_{p}^{1} f(z)^{\beta}=(z-\omega)\left(I_{p}^{0} f(z)^{\beta}\right)+\frac{2(p \beta) \alpha^{\beta}}{(z-\omega)^{p \beta}}=\frac{(p \beta) \alpha^{\beta}}{(z-\omega)^{p \beta}}+\sum_{k=1}^{\infty}(k+p(1-\beta)) a_{k}(\beta)(z-\omega)^{k+p(1-\beta)} \\
I_{p}^{2} f(z)^{\beta}=(z-\omega)\left(I_{p}^{1} f(z)^{\beta}\right)+\frac{2(p \beta)^{2} \alpha^{\beta}}{(z-\omega)^{p \beta}}=\frac{(p \beta)^{2} \alpha^{\beta}}{(z-\omega)^{p \beta}}+\sum_{k=1}^{\infty}(k+p(1-\beta))^{2} a_{k}(\beta)(z-\omega)^{k+p(1-\beta)} \text { an }
\end{gathered}
$$

$\mathrm{d}$ for $n=1,2,3 \ldots$, we can write

$$
I_{p}^{n} f(z)=(z-\omega)\left(I_{p}^{n-1} f(z)^{\beta}\right)^{\prime}+\frac{2(p \beta)^{n} \alpha^{\beta}}{(z-\omega)^{p \beta}}=\frac{(p \beta)^{n} \alpha^{\beta}}{(z-\omega)^{p \beta}}+\sum_{k=1}^{\infty}(k+p(1-\beta))^{n} a_{k}(\beta)(z-\omega)^{k+p(1-\beta)}
$$

\section{Remark:}

(i) In the case $p=\beta=1$, the differential operator $I_{1}^{n}$ was given by Ghanim et al [10].

(ii) In the case $p=\beta=1$ and $\omega=0$, the differential operator $I_{1}^{n}$ was given by Frasin and Darus[6].

\section{Definition:}

The function $f(z)^{\beta} \in S_{\omega}(p, \beta)$ is said to be a member of the class $S T_{\omega}(n, p, \beta, \delta, \psi)$ if it satisfies

$$
\left|\frac{(\psi-1)(z-\omega)\left(I_{p}^{n} f(z)^{\beta}\right)}{I_{p}^{n} f(z)^{\beta}}+(p \beta+\psi)\right|<\left|\frac{(z-\omega)\left(I_{p}^{n} f(z)^{\beta}\right)}{I_{p}^{n} f(z)^{\beta}}+\psi(\delta-1)-p \beta\right|
$$

$$
\left(n \in N_{0}=N \cup 0\right), 0 \leq \alpha<1, p \in N, \beta \in N \text { for some } \delta(0 \leq \delta<1) \text { and } \psi=2,3, . .
$$

It is trivial to see that $S T_{\omega}(0,1,1, \delta, \psi)$ is the class of starlike functions of order $\delta$ and $S T_{\omega}(0,1,1,0, \psi)$ gives the starlike function for all $U$.

Let us now write

$$
S_{\omega}^{*}(n, p, \beta, \delta, \psi)=S T_{\omega}(n, p, \beta, \delta, \psi) \cap S_{\omega}(p, \beta)
$$

where $S_{\omega}(p, \beta)$ is the class of functions of the from (7) that are analytic and univalent in $U$. In the present paper, we shall consider some properties for the classes $S T_{\omega}(n, p, \beta, \delta, \psi)$ and $S_{\omega}^{*}(n, p, \beta, \delta, \psi)$.

\section{Coefficient Inequalities}

Our first result provides a sufficient condition for a function, analytic in $U$ to be in $S T_{\omega}(n, p, \beta, \delta, \psi)$.

Theorem 1: Let the function $f(z)^{\beta}$ be defined by (7) and $\delta(0 \leq \delta<1)$ if

$$
\sum_{k=1}^{\infty}(k+p(1-\beta))^{n}((k+p(1-\beta))+\delta)\left|a_{k}(\beta)\right| \leq \alpha^{\beta}(p \beta)^{n}(p \beta-\delta)
$$

then $f(z)^{\beta} \in S T_{\omega}(n, p, \beta, \delta, \psi)$.

Proof: Suppose the (10) holds true for $0 \leq \beta<1$. Consider the expression 


$$
M\left(f, f^{\prime}\right)=\left|(\psi-1)(z-\omega)\left(I_{p}^{n} f(z)^{\beta}\right)+(\beta p+\psi) I^{n} f(z)^{\beta}\right|-\left|(z-\omega)\left(I_{p}^{n} f(z)^{\beta}\right)+(\psi(\delta-1)-\beta p) I_{p}^{n} f(z)^{\beta}\right| .
$$

That is,

$$
\begin{gathered}
M\left(f, f^{\prime}\right)= \\
\left|\frac{(p \beta(2-\psi)+\psi)(p \beta)^{n} \alpha^{\beta}}{(z-\omega)^{p \beta}}+\sum_{k=1}^{\infty}(k+p(1-\beta))^{n}[\psi(k+p(1-\beta))+p(2 \beta-1)-k+\psi] a_{k}(\beta)(z-\omega)^{k-p(1-\beta)}\right| \\
-\left|\frac{(2 p \beta-\psi(\delta-1))(p \beta)^{n} \alpha^{\beta}}{(z-\omega)^{p \beta}}-\sum_{k=1}^{\infty}(k+p(1-\beta))^{n}[k+p(1-\beta)+\psi(\delta-1)] a_{k}(\beta)(z-\omega)^{k+p(1-\beta)}\right| .
\end{gathered}
$$

Now, for $0<|z-\omega|=r<1$ we have

$$
r^{p \beta} M\left(f, f^{\prime}\right) \leq \sum_{k=1}^{\infty}(k+p(1-\beta))^{n}[\psi(k+p(1-\beta))+\delta]\left|a_{k}(\beta)\right| r^{k+p}-\psi(p \beta-\delta)(p \beta)^{n} \alpha^{\beta} .
$$

The above inequality (11) holds true for all $r(0 \leq r<1)$. Therefore, letting $r \rightarrow 1$ in (11), we obtain

$$
M\left(f, f^{\prime}\right) \leq \sum_{k=1}^{\infty}(k+p(1-\beta))^{n}[\psi(k+p(1-\beta))+\delta]\left|a_{k}(\beta)\right|-\psi(p \beta-\delta)(p \beta)^{n} \alpha^{\beta}
$$

by the hypothesis (10). Hence, it follows that

$$
\left|\frac{(\psi-1)(z-\omega)\left(I_{p}^{n} f(z)^{\beta}\right)}{I_{p}^{n} f(z)^{\beta}}+(p \beta+\psi)\right|<\left|\frac{(z-\omega)\left(I_{p}^{n} f(z)^{\beta}\right)}{I_{p}^{n} f(z)^{\beta}}+\psi(\delta-1)-p \beta\right|
$$

$\left(n \in N_{0}=N \cup 0\right), \quad 0 \leq \alpha<1, \quad p \in N, \beta \in N$ for some $\delta(0 \leq \delta<1)$ and $\psi=2,3, \ldots$. So that $f(z)^{\beta} \in S T_{\omega}(n, p, \beta, \delta, \psi)$. Hence, the theorem is proved.

Corollary 1: Let $n=\delta=0$ in theorem 1, then we have

$$
\sum_{k=1}^{\infty}(k+p(1-\beta))\left|a_{k}(\beta)\right| \leq(p \beta) \alpha^{\beta} .
$$

Corollary 2: Let $n=\delta=0$ and $\alpha=1$ in theorem 1, then we have

$$
\sum_{k=1}^{\infty}(k+p(1-\beta))\left|a_{k}(\beta)\right| \leq p \beta .
$$

Corollary 3: Let $n=\delta=0$ and $p=\beta=1$ in theorem 1, then we have

$$
\sum_{k=1}^{\infty} k\left|a_{k}(1)\right| \leq \alpha
$$

therefore $f$ is starlike univalent in all $z \in U$ with condition $(0 \leq z<1)$.

Corollary 4: Let $n=\delta=0, p=\beta=1$ and $\alpha=1$ in theorem 1, then we have

$$
\sum_{k=1}^{\infty} k\left|a_{k}(1)\right| \leq 1
$$

therefore $f$ is starlike univalent in $U$.

Corollary 5: Let $n=1, \delta=0$, and $p=\beta=1$ in theorem 1, then we have

$$
\sum_{k=1}^{\infty} k^{2}\left|a_{k}(1)\right| \leq \alpha,
$$

therefore $\mathrm{f}$ is convex univalent in all $z \in U$ with condition $(0 \leq z<1)$.

Corollary 6: Let $n=1, \delta=0, p=\beta=1$ and $\alpha=1$ in theorem 1, then we have

$$
\sum_{k=1}^{\infty} k^{2}\left|a_{k}(1)\right| \leq 1
$$

therefore $f$ is convex univalent in $U$. 
Next, we shall give a necessary and sufficient condition for function $f \in S_{\omega}(p, \beta)$ to be in the $\operatorname{class} S_{\omega}^{*}(n, p, \beta, \delta, \psi)$.

Theorem 2: Let the function $f(z)^{\beta}$ be defined by (7) and let $f(z)^{\beta} \in S_{\omega}(p, \beta)$, then $f(z)^{\beta} \in S_{\omega}^{*}(n, p, \beta, \delta, \psi)$ if and only if (10) is satisfied. The result is sharp.

Proof: Assume that $f(z)^{\beta} \in S_{\omega}^{*}(n, p, \beta, \delta, \psi)$. Then

$$
\begin{gathered}
\left|\frac{\frac{(\psi-1)(z-\omega)\left(I_{p}^{n} f(z)^{\beta}\right)}{I_{p}^{n} f(z)^{\beta}}+(p \beta+\psi) \mid}{\frac{(z-\omega)\left(I_{p}^{n} f(z)^{\beta}\right)}{I_{p}^{n} f(z)^{\beta}}+\psi(\delta-1)-p \beta \mid}\right| \\
=\left|\frac{\frac{(p \beta+\psi-p \beta(\psi-1))(p \beta)^{n} \alpha^{\beta}}{(z-\omega)^{p \beta}}+\sum_{k=1}^{\infty}(k+p(1-\beta))^{n} \phi a_{k}(\beta)(z-\omega)^{k+p(1-\beta)}}{\frac{(\psi(\delta-1)-2 p \beta)(p \beta)^{n} \alpha^{\beta}}{(z-\omega)^{p \beta}}+\sum_{k=1}^{\infty}(k+p(1-\beta))^{n} \varphi a_{k}(\beta)(z-\omega)^{k+p(1-\beta)}}\right|<1
\end{gathered}
$$

where

$\phi=[\psi(k+p(1-\beta))+p(2 \beta-1)-k+\psi], \varphi=[k-\psi(1-\delta)+p(1-2 \beta)]$ and $z \in U$.

Since $\mathfrak{R}(z) \leq|z|$ for all $z$, it follows from (12) that

$\mathfrak{R}\left\{\frac{\frac{(p \beta+\psi-p \beta(\psi-1))(p \beta)^{n} \alpha^{\beta}}{(z-\omega)^{p \beta}}+\sum_{k=1}^{\infty}(k+p(1-\beta))^{n} \phi a_{k}(\beta)(z-\omega)^{k+p(1-\beta)}}{\frac{(\psi(\delta-1)-2 p \beta)(p \beta)^{n} \alpha^{\beta}}{(z-\omega)^{p \beta}}+\sum_{k=1}^{\infty}(k+p(1-\beta))^{n} \varphi a_{k}(\beta)(z-\omega)^{k+p(1-\beta)}}\right\}<1$

where

$$
\phi=[\psi(k+p(1-\beta))+p(2 \beta-1)-k+\psi], \varphi=[k-\psi(1-\delta)+p(1-2 \beta)] \text { and } z \in U .
$$

We now choose the values $f(z)^{\beta}$ on the real axis so that $\frac{(z-\omega)\left(I_{p}^{n} f(z)^{\beta}\right)}{I_{p}^{n} f(z)^{\beta}}$ is real. Upon clearing the denominator in (13) and letting $(z-\omega) \rightarrow 1$ through real values, we obtain

$$
\sum_{k=1}^{\infty}(k+p(1-\beta))^{n} \phi a_{k}(\beta) \leq \psi(p \beta-\delta)(p \beta)^{n} \alpha^{\beta}-\sum_{k=1}^{\infty}(k+p(1-\beta))^{n} \varphi a_{k}(\beta)
$$

where $\phi$ and $\varphi$ are as earlier defined.

This implies that

$$
\sum_{k=1}^{\infty}(k+p(1-\beta))^{n}[\psi(k+p(1-\beta)+\delta)] a_{k}(\beta) \leq \psi(p \beta-\delta)(p \beta)^{n} \alpha^{\beta}
$$

which immediately yield the required condition (12).

Our assertion in Theorem 2 is best possible for the functions of the from

$$
\begin{gathered}
f_{k+p(1-\beta)}(z)^{\beta}=\frac{\alpha^{\beta}}{(z-\omega)^{p \beta}}+\frac{(p \beta-\delta)(p \beta)^{n} \alpha^{\beta}}{(k+p(1-\beta))^{n}[k+p(1-\beta)+\delta]}(z-\omega)^{k+p(1-\beta)} \\
\left(k \geq 1, n \in N_{0}, p, \beta \in N, 0 \leq \delta<1,0<\alpha \leq 1\right)
\end{gathered}
$$

Corollary 7: Let the function $f(z)^{\beta}$ be defined by (7) and let $f(z)^{\beta} \in S_{\omega}(p, \beta)$. If $f \in S_{\omega}^{*}(n, p, \beta, \delta, \psi)$, then 


$$
a_{k}(\beta) \leq \frac{(p \beta-\delta)(p \beta)^{n} \alpha^{\beta}}{(k+p(1-\beta))^{n}[k+p(1-\beta)+\delta]} .
$$

The result in (16) is best possible for function $f_{k+p(1-\beta)}(z)^{\beta}$ given by (15).

Now, the distortion property for the functions in the class $S_{\omega}^{*}(n, p, \beta, \delta, \psi)$ is contained in the next Theorem.

Theorem 3: If the function $f(z)^{\beta}$ defined by (7) is in the class $S_{\omega}^{*}(n, p, \beta, \delta, \psi)$, then for $0<|z-\omega|=r<1$, we have

$$
\begin{aligned}
& \frac{\alpha^{\beta}}{r^{p \beta}}-\frac{(p \beta-\delta)(p \beta)^{n} \alpha^{\beta} r^{1+p(1-\beta)}}{(1+p(1-\beta))^{n}[1+p(1-\beta)+\delta]} \leq\left|f(z)^{\beta}\right| \\
& \leq \frac{\alpha^{\beta}}{r^{p \beta}}+\frac{(p \beta-\delta)(p \beta)^{n} \alpha^{\beta} r^{1+p(1-\beta)}}{(1+p(1-\beta))^{n}[1+p(1-\beta)+\delta]}
\end{aligned}
$$

with equality for

and

$$
f_{1}(z)^{\beta}=\frac{\alpha^{\beta}}{(z-\omega)^{p \beta}}+\frac{(p \beta-\delta)(p \beta)^{n} \alpha^{\beta}(z-\omega)^{1+p(1-\beta)}}{(1+p(1-\beta))^{n}[1+p(1-\beta)+\delta]}(z=i r, r)
$$

$$
\begin{aligned}
& \frac{(p \beta) \alpha^{\beta}}{r^{p \beta+1}}-\frac{(p \beta-\delta)(p \beta)^{n} \alpha^{\beta} r^{p(1-\beta)}}{(1+p(1-\beta))^{n-1}[1+p(1-\beta)+\delta]} \leq\left|\left(f(z)^{\beta}\right)\right| \\
& \leq \frac{(p \beta) \alpha^{\beta}}{r^{p \beta+1}}+\frac{(p \beta-\delta)(p \beta)^{n} \alpha^{\beta} r^{p(1-\beta)}}{(1+p(1-\beta))^{n-1}[1+p(1-\beta)+\delta]}
\end{aligned}
$$

with equality for

$$
f_{1}(z)^{\beta}=\frac{\alpha^{\beta}}{(z-\omega)^{p \beta}}+\frac{(p \beta-\delta)(p \beta)^{n} \alpha^{\beta}(z-\omega)^{1+p(1-\beta)}}{(1+p(1-\beta))^{n}[1+p(1-\beta)+\delta]}(z=i r, r)
$$

Proof: Since $S_{\omega}^{*}(n, p, \beta, \delta, \psi)$, theorem 2 readily yields the inequality

$$
\sum_{k=1}^{\infty} a_{k}(\beta) \leq \frac{(p \beta-\delta)(p \beta)^{n} \alpha^{\beta}}{(k+p(1-\beta))^{n}[k+p(1-\beta)+\delta]} .
$$

Thus, for $0<|z-\omega|=r<1$, and making use of (19), we have

and

$$
\left|f(z)^{\beta}\right| \leq \frac{\alpha^{\beta}}{r^{p \beta}}+r^{1+p(1-\beta)} \sum_{k=1}^{\infty}\left|a_{k}(\beta)\right| \leq \frac{\alpha^{\beta}}{r^{p \beta}}+\frac{(p \beta-\delta)(p \beta)^{n} \alpha^{\beta} r^{1+p(1-\beta)}}{(1+p(1-\beta))^{n}[1+p(1-\beta)+\delta]}
$$

$$
\left|f(z)^{\beta}\right| \geq \frac{\alpha^{\beta}}{r^{p \beta}}-r^{1+p(1-\beta)} \sum_{k=1}^{\infty}\left|a_{k}(\beta)\right| \geq \frac{\alpha^{\beta}}{r^{p \beta}}-\frac{(p \beta-\delta)(p \beta)^{n} \alpha^{\beta} r^{1+p(1-\beta)}}{(1+p(1-\beta))^{n}[1+p(1-\beta)+\delta]} .
$$

Also, from theorem 2, it follows that

$$
\sum_{k=1}^{\infty}(k+p(1-\beta)) a_{k}(\beta) \leq \frac{(p \beta-\delta)(p \beta)^{n} \alpha^{\beta} r^{p(1-\beta)}}{(1+p(1-\beta))^{n-1}[1+p(1-\beta)+\delta]} .
$$

Hence,

$$
\left|\left(f(z)^{\beta}\right)\right| \leq \frac{(p \beta) \alpha^{\beta}}{r^{p \beta+1}}+r^{p(1-\beta)} \sum_{k=1}^{\infty}(k+p(1-\beta)) a_{k}(\beta) \leq \frac{(p \beta) \alpha^{\beta}}{r^{p \beta+1}}+\frac{(p \beta-\delta)(p \beta)^{n} \alpha^{\beta} r^{p(1-\beta)}}{(1+p(1-\beta))^{n-1}[1+p(1-\beta)+\delta]} \text { and }
$$




$$
\left|\left(f(z)^{\beta}\right)\right| \geq \frac{(p \beta) \alpha^{\beta}}{r^{p \beta+1}}-r^{p(1-\beta)} \sum_{k=1}^{\infty}(k+p(1-\beta)) a_{k}(\beta) \geq \frac{(p \beta) \alpha^{\beta}}{r^{p \beta+1}}-\frac{(p \beta-\delta)(p \beta)^{n} \alpha^{\beta} r^{p(1-\beta)}}{(1+p(1-\beta))^{n-1}[1+p(1-\beta)+\delta]}
$$

This completes the proof of theorem 3 .

\section{Radii of Closes-to-Convex, Starlikeness and Convexity}

The radii of starlikeness and convexity for the class $S_{\omega}^{*}(n, p, \beta, \delta, \psi)$, is given by the following theorems.

Theorem 4: If the function $f(z)^{\beta}$ defined by (7) is in the class $S_{\omega}^{*}(n, p, \beta, \delta, \psi, \tau)$, then $f(z)^{\beta}$ is close-toconvex of order $\tau(0 \leq \tau<1)$ in $|z-\omega|<r_{1}$, where

$$
r_{1}=r_{1}(n, p, \beta, \delta, \psi, \tau)=\inf _{k}\left\{\frac{(p \beta-\tau)(k+p(1-\beta))^{n}(k+p(1-\beta)+\delta)}{(p \beta-\delta)(k+p(1-\beta))(p \beta)^{n}}\right\}^{\frac{1}{k+p}} .
$$

The result is Sharp for the function $f_{k}(z)^{\beta}$ given by (15).

Proof: It suffices to show that

$$
\left|\frac{\left(f(\beta)^{\beta}\right)}{\alpha^{\beta}(z-\omega)^{-p \beta-1}}+p \beta\right|
$$

for $|z-\omega|<r_{1}$. We have

$$
\begin{gathered}
=\left|\frac{\left(f(\beta)^{\beta}\right)}{\alpha^{\beta}(z-\omega)^{-p \beta-1}}+p \beta\right| \\
=\left|\frac{\sum_{k=1}^{\infty}(k+p(1-\beta)) a_{k}(\beta)(z-\omega)^{k+p}}{\alpha^{\beta}}\right| \leq \frac{\sum_{k=1}^{\infty}(k+p(1-\beta)) a_{k}(\beta)|z-\omega|^{k+p}}{\alpha^{\beta}} .
\end{gathered}
$$

Hence, (23) holds true if

With the help of (10), (24) is true if

$$
\frac{\sum_{k=1}^{\infty}(k+p(1-\beta)) a_{k}(\beta)|z-\omega|^{k+p}}{\alpha^{\beta}(p \beta-\tau)} \leq 1 .
$$

$$
\frac{\sum_{k=1}^{\infty}(k+p(1-\beta)) a_{k}(\beta)|z-\omega|^{k+p}}{\alpha^{\beta}(p \beta-\tau)} \leq \frac{\sum_{k=1}^{\infty}(k+p(1-\beta))^{n}(k+p(1-\beta)+\delta)}{\alpha^{\beta}(p \beta)^{n}(p \beta-\delta)}(k \geq 1)
$$

That is,

$$
|z-\omega| \leq\left\{\frac{(p \beta-\tau)(k+p(1-\beta))^{n}(k+p(1-\beta)+\delta)}{(p \beta-\delta)(k+p(1-\beta))(p \beta)^{n}}\right\}^{\frac{1}{k+p}}
$$

and this completes the proof of theorem 4 .

Theorem 5: If the function $f(z)^{\beta}$ defined by (7) is in the class $S_{\omega}^{*}(n, p, \beta, \delta, \psi, \tau)$, then $f(z)^{\beta}$ is starlike of order $\tau(0 \leq \tau<1)$ in $|z-\omega|<r_{2}$,

where

$r_{2}=r_{2}(n, p, \beta, \delta, \psi, \tau)=\inf _{k}\left\{\frac{(p \beta-\tau)(k+p(1-\beta))^{n}(k+p(1-\beta)+\delta)}{(p \beta-\tau)(k+p(1-\beta)-\tau)(p \beta)^{n}}\right\}^{\frac{1}{k+p}}$.

The result is best possible for the function $f_{k+p(1-\beta)}(z)^{\beta}$ given by (15).

Proof: It suffices to prove that 


$$
\left|\frac{(z-\omega)\left(f(z)^{\beta}\right)}{f(z)^{\beta}}+p \beta\right|
$$

for $|z-\omega|<r_{2}$, We have

$$
\begin{aligned}
& \left|\frac{(z-\omega)\left(f(z)^{\beta}\right)^{\prime}}{f(z)^{\beta}}+p \beta\right|=\left|\frac{\sum_{k=1}^{\infty}(k+p) a_{k}(\beta)(z-\omega)^{k+p(1-\beta)}}{\frac{\alpha^{\beta}}{(z-\omega)^{p \beta}}+\sum_{k=1}^{\infty} a_{k}(\beta)(z-\omega)^{k+p(1-\beta)}}\right| \\
& =\left|\frac{\sum_{k=1}^{\infty}(k+p) a_{k}(\beta)(z-\omega)^{k+p}}{\alpha^{\beta}+\sum_{k=1}^{\infty} a_{k}(\beta)(z-\omega)^{k+p}}\right| \leq \frac{\sum_{k=1}^{\infty}(k+p) a_{k}(\beta)|z-\omega|^{k+p}}{\alpha^{\beta}-\sum_{k=1}^{\infty} a_{k}(\beta)|z-\omega|^{k+p}} .
\end{aligned}
$$

Hence, (29) holds true if

$$
\sum_{k=1}^{\infty}(k+p) a_{k}(\beta)|z-\omega|^{k+p} \leq(p \beta-\tau)\left[\alpha^{\beta}-\sum_{k=1}^{\infty} a_{k}(\beta)|z-\omega|^{k+p}\right]
$$

or

$$
\frac{\sum_{k=1}^{\infty}(k+p(1-\beta)-\tau) a_{k}(\beta)|z-\omega|^{k+p}}{(p \beta-\tau) \alpha^{\beta}} \leq 1
$$

with the aid of (10), (31) is true if

$$
\frac{\sum_{k=1}^{\infty}(k+p(1-\beta)-\tau) a_{k}(\beta)|z-\omega|^{k+p}}{(p \beta-\tau) \alpha^{\beta}} \leq \frac{(k+p(1-\beta))^{n}(k+p(1-\beta)+\delta)}{\alpha^{\beta}(p \beta)^{n}(p \beta-\delta)}(k \geq 1)
$$

which implies that

$$
|z-\omega| \leq\left\{\frac{(p \beta-\tau)(k+p(1-\beta))^{n}(k+p(1-\beta)+\delta)}{(p \beta-\tau)(k+p(1-\beta)-\tau)(p \beta)^{n}}\right\}^{\frac{1}{k+p}}
$$

and this completes the proof of theorem 5 .

Theorem 6: If the function $f(z)^{\beta}$ defined by (7) is in the class $S_{\omega}^{*}(n, p, \beta, \delta, \psi, \tau)$, then $f(z)^{\beta}$ is convex of order $\tau(0 \leq \tau<1)$ in $|z-\omega|<r_{3}$,

where

$$
r_{3}=r_{3}(n, p, \beta, \delta, \psi, \tau)=\inf _{k}\left\{\frac{(p \beta-\tau-1)(k+p(1-\beta))^{n-1}(k+p(1-\beta)+\delta)}{(p \beta-\tau)(k+p(1-\beta)-\tau)(p \beta)^{n}}\right\}^{\frac{1}{k+p}} .
$$

The result is best possible for the function given by (15).

Proof: Using the same technique employed in the proof of theorem 4 and 5, we can show that

$$
\left|\frac{(z-\omega)\left(f(\beta)^{\beta}\right)^{\prime}}{\left(f(\beta)^{\beta}\right)^{i}}+1+p \beta\right| \leq(p \beta-\tau)
$$

for $|z-\omega|<r_{2}$ with the aid of theorem 1. Thus, we have the assertion of theorem 6 . 


\section{Convex Linear Combination}

Our next results involve linear combination of several functions of the type (15).

Theorem 7: Let

$$
f_{p(1-\beta)}(z)^{\beta}=\frac{\alpha^{\beta}}{(z-\omega)^{p \beta}}
$$

and

$$
f_{k+p(1-\beta)}(z)^{\beta}=\frac{\alpha^{\beta}}{(z-\omega)^{p \beta}}+\frac{(p \beta-\delta)(p \beta)^{n} \alpha^{\beta}}{(k+p(1-\beta))^{n}(k+p(1-\beta)+\delta)}(z-\omega)^{k+p(1-\beta)} .
$$

Then, $f(z)^{\beta} \in S_{\omega}(n, p, \beta, \delta, \psi)$ if and only if it can be expressed in the form

$$
f(z)^{\beta}=\sum_{k=0}^{\infty} \Omega_{k+p(1-\beta)} f_{k+p(1-\beta)}(z)^{\beta}
$$

where

$$
\Omega_{k+p(1-\beta)} \geq 0 \text { and } \sum_{k=0}^{\infty} \Omega_{k+p(1-\beta)}=1 .
$$

Proof: From (36), (37) and (38), it is easily seen that

$$
\begin{gathered}
f(z)^{\beta}=\sum_{k=0}^{\infty} \Omega_{k+p(1-\beta)} f_{k+p(1-\beta)}(z)^{\beta} \\
=\frac{\alpha^{\beta}}{(z-\omega)^{p \beta}}+\frac{(p \beta-\delta)(p \beta)^{n} \alpha^{\beta}}{(k+p(1-\beta))^{n}(k+p(1-\beta)+\delta)} \Omega_{k+p(1-\beta)}(z-\omega)^{k+p(1-\beta)} .
\end{gathered}
$$

Since$$
\sum_{k=0}^{\infty} \frac{(k+p(1-\beta))^{n}(k+p(1-\beta)+\delta)}{(p \beta-\delta)^{n}(p \beta)^{n} \alpha^{\beta}} \Omega_{k+p(1-\beta)} \cdot \frac{(p \beta-\delta)^{n}(p \beta)^{n} \alpha^{\beta}}{(k+p(1-\beta))^{n}(k+p(1-\beta)+\delta)}=\sum_{k=1}^{\infty} \Omega_{k+p(1-\beta)}
$$$$
=1-\Omega_{p(1-\beta)} \leq 1 .
$$

It follows from theorem 2 that the function $f(z)^{\beta} \in S_{\omega}^{*}(n, p, \beta, \delta, \psi)$.

Conversely, let us suppose that $f(z)^{\beta} \in S_{\omega}^{*}(n, p, \beta, \delta, \psi)$. Since

$$
a_{k} \leq \frac{(p \beta-\delta)(p \beta)^{n} \alpha^{\beta}}{(k+p(1-\beta))^{n}(k+p(1-\beta)+\delta)}\left(k \geq 1, \quad n \in N_{0}\right),
$$

setting

and

$$
\Omega_{k+p(1-\beta)}=\frac{(k+p(1-\beta))^{n}(k+p(1-\beta)+\delta)}{(p \beta-\delta)(p \beta)^{n} \alpha^{\beta}}\left(k \geq 1, \quad n \in N_{0}\right)
$$

It follows that

$$
\Omega_{p(1-\beta)}=1-\sum_{k=1}^{\infty} \Omega_{k+p(1-\beta)} .
$$

$$
f(z)^{\beta}=\sum_{k=0}^{\infty} \Omega_{k+p(1-\beta)} f_{k+p(1-\beta)}(z)^{\beta} .
$$

This completes the proof of theorem 7 .

Theorem 8: The class $S_{\omega}^{*}(n, p, \beta, \delta, \psi)$ is closed under convex linear combination.

Proof: Let us suppose that the functions $f_{1}(z)^{\beta}$ and $f_{2}(z)^{\beta}$ defined by 


$$
f_{j}(z)^{\beta}=\frac{\alpha^{\beta}}{(z-\omega)^{p \beta}}+\sum_{k=1}^{\infty} a_{k, j}(z-\omega)^{k+p(1-\beta)} \quad(j=1,2 ; z \in U)
$$

are in the class $S_{\omega}^{*}(n, p, \beta, \delta, \psi)$. Also, setting

$$
f(z)^{\beta}=\mu f_{1}(z)^{\beta}+(1-\mu) f_{2}(z)^{\beta}(0 \leq u<1) .
$$

Then, from (40), we can write that

$$
f(z)^{\beta}=\frac{\alpha^{\beta}}{(z-\omega)^{p \beta}}+\sum_{k=1}^{\infty},\left({ }^{\mu} a_{k, 1}+(1-\mu) a_{k, 2}\right) \quad(0 \leq \mu<1 ; z \in U) .
$$

Thus, in view of theorem 2, we can have that

$$
\begin{gathered}
\sum_{k=1}^{\infty}\left[(k-p(1-\beta))^{n}(k-p(1-\beta)+\delta)\right]\left(\mu a_{k, 1}+(1-\mu) a_{k, 2}\right) \\
=\mu \sum_{k=1}^{\infty}(k+p(1-\beta))^{n}(k+p(1-\beta)+\delta)+a_{k, 1}(1-\mu) \sum_{k=1}^{\infty}(k-p(1-\beta))^{n}(k+p(1-\beta)+\delta) a_{k, 2} \\
\leq \mu(p \beta-\delta)(p \beta)^{n} \alpha^{\beta}+(1-\mu)(p \beta-\delta)(p \beta)^{n} \alpha^{\beta}=(p \beta-\delta)(p \beta)^{n} \alpha^{\beta}
\end{gathered}
$$

which shows that $f(z)^{\beta} \in S_{\omega}^{*}(n, p, \beta, \delta, \psi)$ and this complete the proof of the theorem.

\section{References}

[1] M. Acu, S. Owa, On some subclass of univalent functions, J. Inequalitity in Pure and Appl. Math., 6 (2005), 1-6.

[2] M.K. Aouf, On a certain class of meromorphic univalent function with positive coefficient Rend. Math., 7 No. 11 (1991), $209-219$.

[3] N.E. Cho, S.H. Lee, S. Owa, A class of meromorphic univalent function with positive coefficicent, Kobe J. Math., 4 (1987), $43-50$

[4] J. Clunie, On meromorphic schlicht functions, J. London Math. Soc, 34 (1995), 205-216,

[5] P.L. Duren, Univalent functions, Grundlehren Der Mathematischen Wissenschafen, volume 259, Springer-Verlag, Newyork-BerlinHeidelberg-Tokyo (1983).

[6] B.A. Frasin. M. Darus, On certain meromorphic functions with positive coefficients, South East Asian bulletin of Math., 28 (2004), $615-623$.

[7] R.M. Geol, N.S. Sohi, On a class of meromorphic functions, Glas. Mat, Ser. III, 17 No. 37 (1982), 19-28,

[8] A.W. Goodman, on uniformly starlike functions, J. Math Anal. Appl, 155 (1991), 364-370.

[9] A.W. Goodman, On uniformly convex functions, Ann. Polon. Math., 56 (1991), 87-92.

[10] F. Ghanim, M. Darus, S. Sivasubramanian On new subclass of analytic univalent function, Int. J. of Pure and Applied Mathematics, 40, No.3 (2007), 307-319.

[11] S. Kanas, F. Ronning, Uniformly starlike and conver function and other related classes of univalent functions, Ann. Univ. Mariae Curie - Sklodowska Section A, 53 (1999), 95-105

[12] A.T. Oladopo, Coefficient Inequalities and Convolution properties for certain New class of Analytic and Univalent Functions in the unit Disk, Far East Journal of Mathematical Sciences 62, No.2 (2012), 219-232.

[13] B.A. Uralgaddi, C. Somanatha, certain differential operators for meromorphic functions, Houseton J. Math., 17 (1991). $279-284$. 